\title{
Proceedings of the 122nd Meeting of the Society of British Neurological Surgeons, Nottingham, 26-28 May 1993
}

REGIONAL DIFFERENCES IN MANAGING SUBARACHNOID HAEMORRHAGE: THREE YEARS OF AUDIT FROM NEWCASTLE AND NOTTINGHAM

DT Hope, JC Stevenson, V Orpe, I Chambers, AD Mendelow. Queen's Medical Centre, Nottingham and Newcastle General Hospital, Newcastle upon Tyne, UK

All subarachnoid haemorrhage patients (850) admitted to the neurosurgical units of Queen's Medical Centre and Newcastle General Hospital between January 1990 and December 1992 have been audited. The purpose was to look at differing patterns of referral and presentation. Of particular interest were the World Federation of Neurosurgical Societies (WFNS) grades on admission (and on day of surgery), the interval from bleed to admission and from admission to surgery. The total length of stay in each regional unit was audited and the outcome as judged at discharge. The study attempted to identify areas where the quality of care may be improved in the management of subarachnoid haemorrhage. The patients in good grades, WFNS I or II, still showed wide variations in the timing of surgery, possibly reflecting the availability of beds, angiogram facilities, and the number of consultants. Surgeons in Newcastle tended to operate earlier than those in Nottingham although the overall outcome was similar. Delayed surgery was associated with a higher rebleed rate in patients in good grades. Direct comparisons of outcome between surgical units can be misleading unless strict criteria for comparison can be agreed. To measure quality of care, standards must be established and British neurosurgery has, as a specialty, been slow to set these.

MOBILISATION OF ZYGOMA FACILITATES BASILAR ARTERY ANEURYSM SURGERY ATH Casey, D Uttley, D Archer. Atkinson Morley's Hospital and Royal Marsden Hospital, London, UK

An improved understanding of skull base and vascular anatomy and an appreciation of the need to minimise brain retraction has resulted in some ingenious approaches to skull-base lesions. In 1985 the advantages that mobilisation of the zygoma would afford in clipping terminal basilar artery aneurysms were first realised.

Since 1986 this approach has been used in 10 patients. Their mean age was 43 years (range 23-58) with admission in WFNS grade II (range I-V). Surgery was performed via a frontozygomatic approach at an average of 8.4 days (range 1-18) from ictus. $^{2}$ Four patients had multiple aneurysms whose definitive treatment was also possible with this approach. There were two fatalities occurring 48 hours and 72 hours postoperatively which were attributed to brainstem perforator involvement and vasospasm, respectively. The surviving patients all achieved a satisfactory final outcome with one patient having a persistent third-nerve palsy. There were no permanent facial nerve sequelae or significant jawopening difficulties. This approach is therefore encouraging and adds little to the overall length of the operation. It is therefore recommended for the treatment of terminal basilar artery aneurysms.

1 Fujitsu K, Kutawara T. Zygomatic approach for lesions in the interpeduncular cistern. $\mathcal{F}$ Neurosurg 1985;62:340-3

2 Uttley D, Archer DJ, Bell BA. Improved access to lesions of the central skull base by mobilisation of the zygoma: experience with 54 cases. Neurosurgery 1991;28:99-104.

THE PREDICTION OF DELAYED CEREBRAL ISCHAEMIA FOLLOWING SUBARACHNOID HAEMORRHAGE BY MEASURING MIDDLE CEREBRAL ARTERY MEAN FLOW VELOCITY AND MEAN CEREBRAL TRANSIT TIME FC Wong, IR Piper, JD Miller, C Stevenson, M Merrick. University of Edinburgh, Western General Hospital, Edinburgh, UK

The cause of delayed cerebral ischaemia (DCI) following subarachnoid haemorrhage is multifactorial. The measurement of middle cerebral artery mean flow velocity to detect vasospasm combined with the measurement of mean cerebral transit time (MCTT) as an index of cerebral perfusion reserve may predict the onset of DCI. Both methods are relatively inexpensive, can be readily available and can be performed at the bedside.

Middle cerebral artery mean flow velocity has been monitored (on a daily basis if required) by transcranial Doppler sonography (TCD). Vasospasm was defined when the mean flow velocity exceeded $120 \mathrm{~cm} / \mathrm{s}$. MCTT has been measured using a technique developed in Edinburgh based on the first-pass transit of a radiolabelled intravascular tracer injected intravenously and detected as it passes through the cerebral circulation using a standard gamma camera. Normal hemispheric MCTT is between 2.5 and $7 \cdot 7 \mathrm{~s}$ ( $95 \%$ confidence interval) and normal interhemispheric assymmetry does not exceed $0.6 \mathrm{~s}$.

Eighteen patients admitted with subarachnoid haemorrhage have had both MCTT and TCD studies performed. Six patients developed DCI and all six patients had either Doppler-defined vasospasm $(n=4)$ or abnormal MCTT results $(n=5)$ or both $(n=3)$. Localised or unilateral abnormalities matched the clinical neurological deficits of the patients. The combined application of both these tests may be potentially useful in predicting delayed cerebral ischaemia.

CEREBRAL ISCHAEMIC EFFECTS OF ENDOTHELIN AND INDUCED SUBARACHNOID HAEMORRHAGE

AH Huneidi, C Thiemermann, D Drincevic, $F$ Afshar, JR Vane. St Bartholomew's Hospital, London, UK

The development of cerebral ischaemia following the systemic administration of the vasoconstrictor peptides endothelin-1 (ET$1 ; 1 \mathrm{nmol} . \mathrm{kg}^{-1} ; \mathrm{n}=11$ ), big-endothelin (bET; 3 nmol. $\mathrm{kg}^{-1} ; \mathrm{n}=11$ ), or angiotensin-II (A-II; $10 \mathrm{nmol} \cdot \mathrm{kg}^{-1} \cdot \mathrm{min}^{-1} .30 \mathrm{~min} ; \mathrm{n}=8$ ) was compared with a double subarachnoid haemorrhage model in the NZ white rabbit Haemorrhage was induced by an autologous arterial blood injection into the cisterna magna on day 1 (SAH-I; $n=11)$ and on days 1 and 4 (SAH-II; $n=11$ ). Brain histology showed acute neuronal and astrocyte swelling, cytoplasmic vacuolation, pyknosis, and cell loss in the hippocampus (HPC), cerebellum (CBLM) and brain stem (BS) Electron microscopy of the basilar artery endothelium (BA-END) showed vacuolation, mitochondrial loss, and cellular disruption. The table shows the relative incidence as the mean (SD) percentage of ischaemia and vascular endothelial damage in all groups.

\begin{tabular}{lrllll}
\hline Group & $n$ & \multicolumn{1}{l}{$B S$} & $H P C$ & $C B L M$ & $B A-E N D$ \\
\hline SAH-I & 11 & $38(13)$ & $38(13)$ & $38(13)$ & $31(13)$ \\
SAH-II & 11 & $62(13)$ & $69(13)$ & $69(13)$ & $69(13)$ \\
ET-1 & 11 & $55(15)$ & $64(14)$ & $55(15)$ & $64(14)$ \\
B-ET & 11 & $64(14)$ & $72(14)$ & $72(14)$ & $82(12)$ \\
A-II & 8 & $22(5)$ & $25(12)$ & $20(7)$ & $15(6)$ \\
Control & 12 & $8(5)$ & $10(9)$ & $10(9)$ & $10(9)$ \\
\hline
\end{tabular}

It is concluded that ET-1 and b-ET produced cerebral ischaemia with a similar incidence and pattern to ischaemia complicating SAH. Endothelins may thus participate in the pathogenesis of ischaemic complications of subarachnoid haemorrhage, but this still needs further elucidation.

MANAGEMENT AND OUTCOME OF PATIENTS IN POOR NEUROLOGICAL GRADE FOLLOWING SUBARACHNOID HAEMORRHAGE

MG O'Sullivan, C McIntosh, R Sellar, AJW Steers, IR Whittle, JD Miller. Western General Hospital, Edinburgh, UK

The prognosis for patients in poor neurological grades (WFNS grades IV and V) following subarachnoid haemorrhage $(\mathrm{SAH})$ is poor. ${ }^{1} \mathrm{~A}$ favourable outcome was recently reported in $65 \%$ of a selected cohort of 


\begin{tabular}{|c|c|c|c|c|c|c|c|}
\hline \multicolumn{4}{|c|}{ Outcome according to group } & \multicolumn{4}{|c|}{ Outcome according to CT abnormality } \\
\hline $\operatorname{Group}(n)$ & $D$ & $P V S+S D$ & $M D+G$ & $C T$ & $D$ & $P V S+S D$ & $M D+G$ \\
\hline $\begin{array}{l}1(16) \\
2(12) \\
3(34)\end{array}$ & $\begin{array}{r}6 \\
1 \\
19\end{array}$ & $\begin{array}{l}2 \\
1 \\
0\end{array}$ & $\begin{array}{r}8 \\
10 \\
15\end{array}$ & $\begin{array}{l}\text { Haematoma (18) } \\
\text { Hydrocephalus (23) } \\
\text { SAH (21) }\end{array}$ & $\begin{array}{r}12 \\
8 \\
6\end{array}$ & $\begin{array}{l}2 \\
0 \\
1\end{array}$ & $\begin{array}{r}4 \\
15 \\
14\end{array}$ \\
\hline
\end{tabular}

$\mathrm{D}=$ dead; $\mathrm{PVS}=$ persistent vegetative state; $\mathrm{SD}=$ severely disabled; $\mathrm{MD}=$ moderately disabled; $\mathrm{G}=$ good recovery.

patients treated intensively. ${ }^{2}$ The authors' experience with patients in poor grades over the two-year period 1991 to 1992 is reported. The aim of the review is to identify deficiencies in the management protocol.

A patient was defined as being in a poor grade if a Glasgow coma score (GCS) of $\leqslant 12$ was recorded within 24 hours of hospital admission. CT scans were reviewed and the predominant abnormality classified as haematoma, hydrocephalus, or diffuse $\mathrm{SAH}$. Outcome was assessed at three months using the Glasgow outcome scale.

Sixty-two patients were suitable for review. Three groups may be defined according to their clinical course: GCS 13-15 on admission with subsequent deterioration (16 patients); GCS $\leqslant 12$ on admission with subsequent improvement (12 patients); and GCS $\leqslant 12$ without improvement, or with improvement following intervention (34 patients). The groups may be further subdivided according to the CT features. The table shows the management and outcome.

Patients in a poor neurological grade following $\mathrm{SAH}$ are a heterogenous group, and urgent CT scanning is required to define the cause of the depressed level of consciousness. Hydrocephalus should be treated intensively. The prognosis for patients in a poor neurological grade due to haematoma is grave and the role of emergency surgery has yet to be defined.

1 Ljundgren B, Soveland H, Brandt L, et al. Early operation and outcome in aneurysmal SAH. $\mathcal{f}$ Neurosurg 1985;62:547-51

2 Bailes J, Spetzler R, Hadley $M$, et al. Management morbidity and mortality of poor grade aneurysm patients. $\mathcal{f}$ Neurosurg $1990 ; 72: 559-66$

RECOGNITION AND MANAGEMENT OF ANTERIOR THORACIC SPINAL CORD HERNIA B D White, I Holland, T Jaspan, JL Firth. University Hospital, Nottingham, UK

Anterior spinal hernia is a rare cause of maturity onset, progressive, thoracic cord dysfunction which has only recently been recognised, with four cases appearing in published reports since 1991 . The patient is typically middle-aged with a history of progressive, mid-thoracic, anterior hemicord syndrome manifesting initially as hemianalgesia below the affected segment with contralateral crural spasticity. Untreated this develops into an asymmetric spastic paraparesis with sparing of dorsal column sensation.

CT myelography demonstrates a focally narrowed thoracic cord kinked and paradoxically drawn towards what appears to be an extradural mass. MRI shows the narrowed, kinked cord closely applied to a vertebral body, and may demonstrate the cord hernia. At operation the cord is found to be morphologically normal but prolapsed and incarcerated in a ventrolateral dural dehiscence. The pathogenesis of the underlying dural defect is unknown, but several hypotheses have been advanced. The syndrome is characteristic, the radiological findings, though subtle, are also typical and surgical repair is easily effected. This condition is probably more common than currently appreciated and should be considered in all patients presenting with progressive thoracic cord dysfunction.

SUBOCCIPITAL TRANSCONDYLAR APPROACH TO THE CLIVUS AND CRANIOCERVICAL JUNCTION

G Neil-Dwyer, DA Lang, F Iannotti. Southampton University Hospital, Southampton, UK

Access to the anterioi rim of the foramen magnum, craniocervical junction, clivus, and front of the lower brainstem can be obtained by using an anterior, lateral or posterolateral approach. The access used should provide good visualisation of the pathology and the surrounding anatomical structures with minimal retraction of neural structures. Ideally the skull should be entered and anatomical routes followed in such a way that there is a short, straight line between the surgeon and the lesion. The preferred approach to this region depends on the exact site of the lesion, the nature of the pathology, and the aim of the operation. Of particular importance is the superior extent of the lesion, its relationship to the neuraxis and its position relative to the midline.

The authors' experience of the transcondylar suboccipital approach is reported, which has been used in 12 patients to remove lesions related to the anterior rim of the foramen magnum, the lateral clivus, the front of the lower brainstem, and for access to the vertebrobasilar circulation. There are limitations imposed both by the location of the pathology and the nature of the lesion, and there is a need for flexibility and combined anterior and transcondylar suboccipital approaches in selected cases.

ODONTOID FRACTURES IN ELDERLY PATIENTS R Viswanathan, MG O'Sullivan, J McEachan, T Russell. Western General Hospital, Edinburgh, UK

Reports detailing cervical spinal injury in patients $\geqslant 65$ years are rare. ${ }^{1}$ A retrospective survey is reported of patients of 65 years or older with odontoid fractures managed in our department over the six-year period to December 1992.

Fifteen patients had isolated odontoid fractures and four suffered injury at multiple levels including the odontoid process (these were excluded from analysis). A fractured odontoid process was found in 50\% of patients with cervical injury in this age group. The median age of the patients was
78 years (range 65-86). Injury occurred as a result of a fall in 12 cases and a road traffic accident in three cases. Type II fractures were seen in 10 patients. Type III in four patients, and Type $I$ in one patient. Two patients had a mild neurological deficit. Nine patients underwent internal stabilisation and six patients were treated with a cervical collar. One patient died and a further patient suffered a monoparesis as a result of surgery. All patients had follow-up assessments that included flexion/extension views of the cervical spine. The median duration of follow up was 38 months (range 11-50 months). All surviving patients were independent with no evidence of instability.

It is concluded that odontoid fractures ccount for a significant proportion of cervical spinal injuries in patients aged 65 years or more, and surgical stabilisation is relatively safe. Type III fractures may be successfully treated with a cervical collar until bony fusion occurs.

1 Pepin JW, Bourne RB, Hawkins RJ. Odontoid fractures, with special reference to the elderly patient. Clin Orthop 1985;193: 178-83.

THE ROLE OF EMERGENCY SURGERY IN MALIGNANT SPINAL EXTRADURAL

COMPRESSION: ASSESSMENT OF FUNCTIONAL OUTCOME

JK Harris, JC Sutcliffe, N Robinson, ES Watkins. Royal London Hospital, London, UK

Delay in reaching neurosurgical care in cases of malignant spinal compression is well known, ${ }^{1}$ but the action taken subsequently by the neurosurgeon is less clearly documented. In the presence of such initial delays should emergency surgical intervention be considered necessary? In this retrospective analysis of functional outcome in 81 patients requiring 84 operations for compressive, malignant, extradural lesions between 1982 and 1991, an assessment of the role of emergency surgery was made independently by physiotherapists and medical staff, with regard to maintaining or improving continence and mobility.

Overall $70 \%$ of patients retained or regained their mobility and $80 \%$ of these were continent postoperatively. Emergency surgery was performed in $65 \%$ cases, and $61.5 \%$ of these experienced improvement, compared with only $25 \%$ of the elective surgery group, pathology in the two groups being similar. Even among those incontinent and immobile, $47 \%$ could walk postoperatively, of whom $76 \%$ had emergency surgery.

The findings suggest that despite initial delays in referral, and even if the patient is incontinent and immobile, emergency spinal decompression is justified.

1 Maurice-Williams RS, Richardson PL. Spina cord compression: delay in the diagnosis and referral of a common neurosurgical emergency. $\mathrm{Br} \mathcal{F}$ Neurosurg 1988;2:55-60.

STRESS AND HYPOTHALAMIC-PITUITARY BLOOD FLOW IN THE RAT

CJ Gerber, T Loughlin, EW Hillhouse, PD Lees. Wessex Neurological Centre, Southampton, and University of Newcastle, Newcastle upon Tyne, UK

Hypothalamic-pituitary portal blood flow controls the anterior pituitary and may 
therefore modulate neuroendocrine function. To test this hypothesis, a new mathematical model was developed and validated in the rat, overcoming the problems of the portal system to allow the measurement of pituitary blood flow using ${ }^{14} \mathrm{C}$-iodoantipyrine autoradiography. ${ }^{1}$ A minimally stressed rat model was also developed, using chronic arterial and venous cannulation with repeated handling to evaluate and overcome the effects of surgical stress which is known to activate the hypothalamicpituitary-adrenal axis. Results show significant elevation of plasma corticosterone and corticotropin under anaesthesia (mean (SE) $536(54) \mathrm{ng} / \mathrm{ml}, 822(159) \mathrm{pg} / \mathrm{ml}$ respectively); at one hour $(585(71) \mathrm{ng} / \mathrm{ml}$, $768(97) \mathrm{pg} / \mathrm{ml}$ ) and one day (441(116) ng/ml, 768(97)pg/ml) compared with control non-cannulated animals (30(3) $\mathrm{ng} / \mathrm{ml}, 460(83) \mathrm{pg} / \mathrm{ml}) \quad(\mathrm{p}=0.01)$. Both return to normal at three days $(25 \cdot 25(0 \cdot 2) \mathrm{ng} / \mathrm{ml}, 572(155) \mathrm{pg} / \mathrm{ml})$. Blood flow was significantly higher at three days in the hypothalamus $(2 \cdot 7(0.48) \mathrm{ml} / \mathrm{g}$ per minute) and anterior pituitary $(1.73(0.2) \mathrm{ml} / \mathrm{g}$ per minute) compared with anaesthetised animals $(1.53(0.36) \mathrm{ml} / \mathrm{g}$ per minute, $0.45(0.05) \mathrm{ml} / \mathrm{g}$ per minute) ( $p=0.05$ ).

These results show that anaesthetised rats and those in acute recovery show stress which resolves by three days. The stressed state is associated with lower blood flow rates in the hypothalamus and pituitary gland than the unstressed state. This suggests that there is a opportunity at three days, when stress is minimised, when the interrelationship between neuroendocrine blood flow and function may be evaluated.

1 Lees PD, Lynch DT, Richards HK, et al $\exists$ Cereb Blood Flow Metab 1992;12:128-38.

CELL CULTURE OF PITUITARY ADENOMAS: ROLE IN CLINICAL MANAGEMENT

S Chatteriee, $\mathbf{R}$ Jeffreys, $\mathbf{P}$ Foy, $\mathbf{S}$ Atkins, $M$ White, L Hipkin, J McKenzie. University of Liverpool, Liverpool, UK

Human pituitary adenomas have been previously classified according to histology, immunocytochemistry, S-phase fraction and their hormone production in vivo. This study looks at categorisation on the basis of cell culture and hormone production in vitro.

The clinical and laboratory details of 50 patients with pituitary adenoma presenting to the Liverpool Neurosurgical Department between 1989 and 1992 were reviewed. All cell cultures were performed using an Isocoves-modified Delbeccos medium with $10 \%$ fetal calf serum. Following successful growth of the cells under special conditions, hormone analysis was performed by radioimmunoassay.

Clinically 14 patients presented with acromegaly, five had obvious features of prolactinomas, two were Cushingoid, three presented with features of panhypopituitarism, and 26 were thought to be functionless before any endocrine evaluation There appeared to be a better correlation of preoperative hormone levels with cellculture data rather than with immunohistochemistry, and this was particularly apparent in patients with acromegaly. In a significant number of cases, the adenoma thought to be producing only growth hormone or prolactin was found, in culture, to be capable of producing both these hormones. In prolactinomas, hormone levels in vitro may be an indicator of prognosis, the larger and more extensive tumours producing higher levels.

AN AUDIT OF THE DIRECT TRANSNASAL TRANSSPHENOIDAL APPROACH TO THE PITUITARY FOSSA

RS Cooke, RAC Jones. Hope Hospital, Salford, UK.

Several routes may be used to gain access to the sphenoid sinus in transsphenoidal pituitary surgery. The direct transnasal approach has been used in this unit since 1987. An audit of this technique is reported in 48 patients who have had 52 transnasa pituitary procedures performed.

Over $80 \%$ of patients presented with an endocrine syndrome. All underwent direct coronal CT. The mean tumour diameter was $17 \mathrm{~mm}$ and $25(52 \%)$ had extrasella extension. The surgical aim (biopsy, debulking or total removal), was achieved in $42(87 \cdot 5 \%)$ cases; in six cases the procedure was aborted due to venous haemorrhage (four patients) or anatomical difficulties (two patients). In these last two cases, a successful re-exploration was made after repeat CT. The mean theatre time was 62 minutes. Complications (diabetes insipidus, cerebrospinal fluid rhinorrhoea, or visual deterioration) occurred after eight procedures $(15.4 \%)$ and were not directly attributable to the transnasal route Postoperative stay averaged three days for uncomplicated cases. No nasal complications have occurred.

Other approaches to the sphenoid' sinus involve difficult dissection through ethmoid air cells, or along the nasal septal submucous plane; the Direct Transnasal approach is straightforward, quick, and does not require laborious dissection of nasal mucosa or removal of septal cartilage. The complication rate is similar to other transsphenoidal series, ${ }^{1}$ but the authors have no encountered any nasal, septal, or dental complications, which can occur with the other approaches.

1 Black P, Zervas N, Guillermo C. Incidence and management of complications of transsphenoidal operation for pituitary adenomas. Neurosurgery 1987;20:920-4.

TRANSSPHENOIDAL SURGERY VERSUS OCTREOTIDE TREATMENT FOR ACROMEGALY F Afshar, AHS Huneidi, TPD Blackburn, JH Wass. St Bartholomew's Hospital, London, UK

Octreotide, a synthetic analogue of somatostatin, has been used recently for the treatment of acromegaly. This prospective study compares octreotide with transsphenoidal surgery in 33 patients (17 men, 16 women mean age 45 years, range 21-64). They were allocated for either octreotide $(n=17$; 8 men) or surgery ( $n=16 ; 8$ men) treatment according to their visual signs. All had pituitary tumours proven by $\mathrm{CT}$, ranging from moderate to large size $(10-30 \mathrm{~mm}$ in their widest diameter). Fifteen patients had intrasellar tumours (meso-adenomas), and 18 had extrasellar extension (macro-adenomas). Mean (SE) growth hormone (GH) levels were 160 (70) $\mathrm{mU}^{-\mathrm{l}^{-1}}$ and 136 (25) $\mathrm{mU} . \mathrm{l}^{-1}$ for the surgery (control) and the octreotide-treated groups respectively.

Octreotide (300-600 $\mu \mathrm{g} /$ day) was given subcutaneously for a period of 2-18 month (mean (SE). 11 (1)). GH levels were reduced to $28.7(6.5) \mathrm{mU} .1^{-1}$. Seven patients showed a minor degree of tumour shrinkage. Subsequently, all the octreotide group patients, except one, underwent surgery. Octreotide reduced the $\mathrm{GH}$ levels by $74 \%$, but did not produce a significant change in the tumour size. Surgery, however, reduced GH levels by $74.5 \%$, and the tumour size by more than $95 \%$.

It is concluded that surgery should be the first line of treatment for GH-producing pituitary adenomas; octreotide and radiotherapy can be reserved as postsurgical adjuvant therapy.

MRI CISTERNOGRAPHY AND LOCALISATION OF CEREBROSPINAL FLUID FISTULAE

MS Eljamel, CN Pidgeon, J Toland, J Phillips, A O'Dwyer. Richmond Institute for Neurology and Neurosurgery, Dublin, Eire

Accurate localisation of cerebrospinal fluid (CSF) fistulae has always been a difficult problem for both the neurosurgeon and neuroradiologist. Several techniques have been advocated over the years with variable success. Precise localisation of the site of CSF leakage not only makes planning of surgery easier, but it also increases the chances of successful dural repair and eliminates negative exploration. Plain skull radiography and complex motion tomography have been superseded by radioisotope cisternography (RIC) and, more recently, by metrizamide CT cisternography (MCTC). However, both RIC and MCTC are invasive, carry risks to the patient and cannot be performed in the presence of intracranial mass lesions. They are time consuming and insensitive, when the CSF leakage is inactive. Furthermore, patients have to be detained in hospital to carry out such investigations. Because CSF gives a high signal on T2-weighted MRI images and MRI can demonstrate the cranial anatomy exquisitely, this is a potentially very valuable localising technique for CSF fistulae.

The aim of this study was to evaluate MRI cisternography in localising the site of inactive CSF leakage. Ten patients with inactive traumatic CSF fistulae were studied using T2-weighted MRI, whose age varied from 11 to 60 years and the female:male ratio was 2:8. Three had Le Forte III fractures and two had petrous bone fractures. Half the patients had meningitis, three of which were recurrent pneumonococcal meningitis. MRI cisternography was performed in all cases and the site of the CSF fistula was demonstrated in the ethmoid sinus (five), frontal sinus (three), cribriform plate (two), sphenoid sinus (one) and the middle ear complex (two). All patients were explored surgically, the sites of CSF fistulae were confirmed and repaired intradurally in each case, using fibrin glue and a pericranial graft.

Although this series is still relatively small, MRI cisternography is safe, carries no risk to the patients, takes a relatively short time to perform and shows the site of the CSF fistula in all cases. MRI cisternography should be the preferred method for localising CSF fistulae, particularly in the absence of active CSF leakage. 
TRANSCRANIAL DOPPLER ULTRASONOGRAPHY AND USE OF A STANDARDISED PULSATILITY INDEX IN THE ASSESSMENT OF PATIENTS WITH HEAD INJURIES

PJ Kirkpatrick, M Czosnyka, V Iyer, $\mathrm{H}$ Whitehouse, P Smielewski, E Guazzo, JD Pickard, Cambridge University, Cambridge, UK

Measurement of middle cerebral artery mean flow velocities (FV) using non-invasive transcranial Doppler ultrasonography has yet to contribute significantly to the routine management of patients with head injuries. Calculation of the dimensionless pulsatility index (PI $\left.=F_{\text {emplitude }} / F_{\text {mean }}\right)$ provides a warning of reduced cerebral perfusion pressure (CPP) in these patients. ${ }^{1}$ In this study, serial transcranial Doppler ultrasonography measurements of FV were undertaken in 21 ventilated patients with diffuse head injury in whom continuous measurements of arterial blood pressure (ABP) and intracranial pressure were acquired allowing coordinated waveform analysis at different values of CPP. The relationship between CPP and mean FV showed a poorly defined autoregulatory threshold at a CPP of approximately $50 \mathrm{~mm}$ $\mathrm{Hg}$. The PI increased gradually, however, as CPP fell below $75 \mathrm{mmHg}$, and more abruptly as CPP fell below $50 \mathrm{mmHg}$. Averaged FV amplitude and ABP amplitude were strongly correlated $(r=0.578$; $\mathrm{p}<0.0001$ ), prompting the calculation of a standardised pulsatility index (SPI = PI/ABP amplitude $_{\text {). As CPP fell below } 70}$ $\mathrm{mmHg}$, the SPI increased more uniformly and at a greater rate than the PI, and showed a more dramatic increase at the autoregulatory threshold. Further, analysis of variance indicated a closer relationship between the SPI and CPP than for the PI. It is concluded that estimation of SPI provides a more accurate index of falling CPP, and may help to define a potential therapeutic opportunity for patients with head injuries.

\section{Chan, et al. $\mathcal{f}$ Neurosurg 1992;77:55-61.}

AUDIT OF TRANSFER OF NEUROSURGICAL PATIENTS

KS El-Shunnar, L Chadwick, G Gill. Oldchurch Hospital, Romford, UK

Neurosurgical patients are often transferred out of normal working hours. Some are mechanically ventilated during transfer and others are critically ill. Several investigators have shown that critically ill patients can suffer secondary insults as a result of transfers both within ${ }^{1}$ and between ${ }^{2}$ hospitals. Eventual mortality in critically ill patients transported between hospitals can be as high as $28 \%$.

Assessment of these patients and the decision to transfer them is often left to junior medical staff inexperienced in dealing with acute neurological problems. An audit of referrals to a regional neurosurgical unit was carried out over a period of 3.5 months. Of 136 patients referred, 26 (19\%) suffered trauma and $42(30 \cdot 8 \%)$ suffered from subarachnoid or intracerebral haemorrhage. A total of $54(39 \cdot 7 \%)$ were referred after hours. A senior house officer was the most senior doctor to assess the patient in 36 $(26.5 \%)$ cases. In $16(11.8 \%)$ cases, a senior house officer was the most senior doctor to know about the patient.
The consultant on call saw the patient in $44(32.3 \%)$ cases and knew about the patient in $66(48.5 \%)$ cases. These figures clearly demonstrate the need for more senior involvement in the management of neurosurgical patients.

1 Andrews PJD, Piper IR, Dearden NM, et al. Secondary insults during intrahospital transport of head-injured patients. Lancet 1990, 335:327-30.

2 Reeve WG, Runcie CJ, Reidy J, et al. Current practice in transferring critically ill patients among hospitals in the west of Scotland. $B M \mathcal{F}$ 1990;300:85-7.

THE OUTCOME OF HEAD INJURIES RESCUED BY A HELICOPTER EMERGENCY MEDICAI SERVICE IN A METROPOLIS

JN Wilden, JC Sutcliffe, R McAvinchey, CJC Kirk, H Hetherington, SE Turvey, A Wilson, IG Wylie, ES Watkins. Royal London Hospital, London, UK

A total of 112 patients with head injuries were rescued by a doctor with advanced training in resuscitative techniques and transported to the Royal London Hospital by helicopter. The service is available to a population of 14 million.

Before reaching the hospital, Glasgow coma score, pulse rate, blood pressure, and pulse oximetry were recorded. In hospital, CT of the brain was performed and the types of injury classified according to the Traumatic Coma Data Bank. ${ }^{1}$ Neurosurgical treatment was started, and outcome categorised according to the Glasgow outcome scale.

At the accident scene, the mean Glasgow Coma Scale was 7, pulse oximetry was reduced in $35 \%$ of cases and $60 \%$ of patients were ventilated. Average time from injury to helicopter arrival was 24 minutes.

Preliminary results demonstrate that $90 \%$ of patients sustaining a diffuse I head injury and $60 \%$ a diffuse II injury achieved "independence" compared with $61 \%$ and $35 \%$ respectively, in the Traumatic Coma Data Bank. Reasons for these significantly different results $(p=0.001)$ were discussed in relation to the methods of measuring severity, type, and outcome of head injury. Definitions of "rapid resuscitation" and "early definitive neurosurgical care" were proposed.

1 Marshall L, Bowers Marshall S, Klauber MR, et al. A new classification of head injury
based on computerized tomography. 7 Neurosurg 1991;75:S14-20.

CEREBELIAR ASTROCYTOMA OF CHILDHOOD: LONG-TERM FOLLOW-UP

$S$ Sgouros, PW Fineron, AD Hockley. Queen Elizabeth Hospital, Birmingham, UK

Cerebellar astrocytoma of childhood has always been regarded as a benign tumour. Certain issues still remain controversialfor example, the role of radiotherapy as an adjuvant to surgery, the management of the cyst wall in cystic tumours, and what are significant adverse prognostic factors. To address these questions, the experience in Birmingham from 1959 to 1991 was reviewed. The records of 97 patients aged $0-14$ years were studied and the histological material assessed independently.

Following clinical and statistical analysis the following conclusions were reached: postoperative radiotherapy does not improve survival, but may actually predispose to malignant transformation; excision of the cyst wall does not enhance survival; and brainstem involvement is the most adverse prognostic factor. Implications on management are discussed.

INTRACRANIAL TUMOURS IN THE ELDERLY: THE EFFECT OF AGE ON THE OUTCOME OF FIRST-TIME SURGERY OF MENINGIOMAS RS Maurice-Williams, ND Kitchen. Royal Free Hospital, London, UK

The effect of age is reported on the outcome of first-time surgery for intracrania meningiomas carried out over 14 years. Patients, reviewed retrospectively, were divided into three age groups: young (up to 44 years, 38 patients); middle-aged (45-64, 60 patients); and elderly ( 65 and over, maximum age 86,46 patients). There were no differences between the groups as regards the sizes or positions of the tumours, except that there were no suprasellar meningiomas in the elderly group. Overall surgical mortality was $2 \cdot 7 \%$. Postoperative complications increased with age $(16,23,30 \%$ respectively) but most of these complications had no lasting effects. Postoperative intracranial bleeding requiring re-operation was common in elderly patients, occurring in $20 \%$ (compared with none in the youngest group), perhaps a reflection of cerebral atrophy leading to a postoperative dead space. Outcome was assessed at the first follow-up appointment 4-6 months after discharge from neurosurgical care. By then, all of the younger patients, $88 \%$ of those who were middle-aged, and $83 \%$ of the elderly were in Glasgow outcome score grade V. A total of $89 \%$ of the elderly patients showed clear improvement compared with their preoperative condition, $2 \%$ were unchanged, and $9 \%$ were worse or had died. Macroscopic total removal of the tumour had been achieved in a greater proportion of the elderly patients $(86 \%)$ than younger patients. The intellectual deterioration apparent in more than half of the elderly patients had recovered fully by the time of first follow-up in $80 \%$. It is concluded that age has little effect on the prospects of success after removal of an intracranial meningioma.

MANAGEMENT OF TUMOURS OF THE PINEAI REGION

L Symon, S Tomita. The National Hospital, London, UK

Management of tumours of the pinea region remains, to some extent, controversial. Diagnosis may be made following imaging studies, with evidence of spread along the habenula region, the presence of seeding in other parts of the ventricular system, indicative of germinoma, or on examination of the ventricular or lumbar cerebrospinal fluid (CSF) for exfoliated cells. Markers of $\alpha$-fetoprotein or human chorionic gonadotrophin in the CSF and blood also may be helpful. In recent years, however, there has been a re-awakened interest in direct surgery for tumours of the pineal region.

In the past 15 years, 12 of 26 pineal tumours have been radically excised by the direct occipital transtentorial approach (four malignant teratomas, two germino- 
mas, two pineoblastomas, one astrocytic tumour, two metastatic tumours, and one ependymoma of the parapineal region). Pineal tumours have been identified in three other cases, all germinomas, by biopsy. Eleven other tumours, three of which were clearly metastatic, were managed by CSF shunting only without positive identification. There has been no operative mortality in radical removal, and symptom-free survival over three years has been noted in the cases of germinoma and in three of the four cases of teratoma in the current group.

HIGH-FREQUENCY EYE TREMOR IN THE ASSESSMENT OF THE COMATOSE PATIENT C Bolger, D Coakley, J Philips, N Sheahan, J Malone. Beaumont Hospital, Dublin, Eire

High-frequency eye tremor or ocular microtremor (OMT) is a constant, physiological, high-frequency tremor of the eye. It is caused by the constant tonic input to brainstem oculomotor centres. ${ }^{1}$ It has been postulated that OMT could be a useful indicator of brainstem dysfunction, particularly in the comatose patient.

The authors have developed a highly portable and accurate method of recording OMT. ${ }^{2}$ Records are obtained at the patient's bedside, each recording taking about 10 minutes. Thirty comatose patients were selected from serial admissions to the neurosurgical intensive therapy unit and compared with 30 age-matched, normal controls. All records were analysed independent of clinical data. The results demonstrate that OMT frequency is significantly reduced in the comatose group $(\mathrm{p}<$ 0.001 ). The frequency correlates positively with the clinical state of the patient. Serial recordings demonstrate that a change in clinical status is reflected in a corresponding change in OMT frequency. A low initial frequency, on admission to intensive therapy, is an indicator of poor prognosis.

1 Coakley D. Minute eye movement and brain stem function, CRC Press, Florida, 1983.

2 Bolger C, Sheehan N, Coakley D, Malone J. High frequency eye tremor: reliability of measurement. Clin Phys Physiol Measurement 1992;13:151-9.

3-D ORBITAL RECONSTRUCTION AFTER

TUMOUR ABLATION

DA Lang, G Neil-Dwyer, BT Evans. Southampton University Hospital, Southampton, UK

Benign lesions, including meningioma en plaque, producing globe displacement, visual deterioration, and facial deformity are a notoriously difficult neurosurgical challenge. Following radical resection of benign tumours in the orbit or anterior cranial fossa, immediate orbital reconstruction is mandatory. To obtain a good cosmetic and functional reconstruction, a three-dimensional appreciation of the orbital anatomy is important so that the orbital volume, margins and bony partitions can be accurately replaced. Rigid fixation is of paramount importance. The authors believe that extensive tumour removal from around the orbit requires a multidisciplinary team and a flexible surgical approach. In 10 patients, including two children with such lesions, these concepts were illustrated.
ORBITAL SURGERY: TECHNICAL ASPECTS AND COMPLICATION AVOIDANCE

IR Whittle. Western General Hospital, Edinburgh, UK

Retrobulbar orbital fossa microsurgery is infrequently performed by most neurosurgeons. Indications for such surgery include dysthyroid eye disease, orbital fossa masses, trauma, and various optic neuropathies. Many of the potential complications of such surgery are unique to the region. The author's experience with 55 cases of orbital surgery is presented with particular emphasis on surgical technique and approaches, potential pitfalls, and occurrence and avoidance of complications.

Indications for surgery included dysthyroid eye disease (29) orbital masses (21 including six meningioma, five haemangioma, two neurofibroma, and one each of lymphoma, teratoma, dermoid cyst, orbital varix, aesthesioneuroblastoma, pseudotumour, adenoid cystic carcinoma, and lacrimal lymphoepithelial hyperplasia), trauma (two) and optic nerve dural fenestration (three). Surgical approaches used included lateral orbitotomy (38), low frontal craniotomy (13), pterional craniotomy (3) and fronto-orbito-zygomatic osteotomy in one case. Patients with dysthyroid eye disease had decompressive superior, posterior, and lateral orbitotomies performed through a small superolateral eyebrow incision. Although short-term or long-term relief of symptoms was unsatisfactory in six eyes, this was unrelated to technical aspects of the surgery. The remaining patients experienced a median $5 \mathrm{~mm}$ globe recession, had improved visual actuity and visual fields, less orbital pain, improved ocular motility, and lower steroid requirements. Excision of orbital masses was generally uncomplicated but particular problems and dilemmas arose with optic nerve sheath meningioma (sacrifice of the optic nerve when some vision is preserved, lateral versus subfrontal approach), patients in whom a second orbital operation was being performed (due to intense scarring in the retroconal tissues) and in one case of retrobulbar lymphoma (iatrogenic monocular blindness). Although optic nerve sheath fenestration is technically simple to perform and led to resolution of papilloedema in all three cases, in none did visual acuity improve. Overall there were no infections and morbidity was low.

TITANIUM CRANIOPLASTY: POST-OPERATIVE RADIOLOGY

CL Chandler, DJ Archer, D Uttley. Atkinson Morley's Hospital, London and Royal Marsden Hospital, London, UK

Titanium cranioplasty has been used in the authors' units since 1988 for the reconstruction of a wide range of cranial defects. Titanium is still regarded by many as less satisfactory than acrylic for the purpose of cranioplasty. It is biologically and chemically inert, light in weight, malleable, and very strong. Most importantly, it is a nonferrous metal, weakly paramagnetic, and relatively radiolucent. ${ }^{12}$

The lack of clinically significant image degradation on MRI, and CT, plain radiography, and contrast cisternography has been demonstrated.

On the basis of its strength, biocompatibility, and excellent handling characteristics, allied to its suitability for all postoperative imaging techniques, it is concluded that titanium plate is the material of choice for cranioplasty.

1 Blake GB, Macfarlane MR, Hinton JW. Titanium in reconstructive surgery of the skull and face. Br $\mathcal{F}$ Plastic Surg 1990;43 528-35.

2 Gordon DS, Blair GAS. Titanium cranioplasty. $B M F$ 1974;2:478.

THE EFFECT OF EXOGENOUS CALCITONIN GENE-RELATED PEPTIDE ON FOCAL CEREBRAL ISCHAEMIC INJURY IN RATS

JP Holland, SG Sydserff, BA Bell Atkinson Morley's Hospital, London, UK

Calcitonin gene-related peptide (CGRP) is a potent endogenous vasodilator existing as a neuropeptide in the trigeminocerebrovascular system. ${ }^{1}$ It is believed that the (nociceptive) depolarisation of these nerves in the event of a subarachnoid haemorrhage or migraine causes the antidromic release of CGRP in an attempt to restore normal cerebrovascular tone. ${ }^{2}$

The effect of intravenous CGRP was studied in a rat model of middle cerebral artery (MCA) occlusion, observing the effects on cerebral blood flow and ischaemic neuronal injury. The results show that an infusion of intravenous CGRP started one hour before MCA occlusion, produces an improved cerebral blood flow in the ischaemic hemisphere (control: mean (SD) $13.3(1.8) \mathrm{ml} / 100 \mathrm{~g}$ per minute, CGRP: $32.3(2 \cdot 1) \mathrm{ml} / 100 \mathrm{~g}$ per minute) and reduces the volume of ischaemic neuronal injury by $58 \%(n=10)$. A CGRP infusion started one hour after MCA occlusion similarly maintains cerebral blood flow and reduces the volume of ischaemic neuronal injury by $38 \%(n=5)$.

The results demonstrate that exogenous CGRP improves the ischaemic microenvironment and reduces the volume of ischaemic neuronal injury. The clinical benefit of this peptide to combat the effects of a predicted ischaemic insult, such as may occur following subarachnoid haemorrhage or during carotid endarterectomy, needs to be re-examined.

1 Edvinsson $\mathrm{L}$, et al. Peptide containing nerve fibres in human cerebral arteries: immunocytochemistry, radioimmunoassay and cytochemistry, radioimmunoassay and
in-vitro pharmacology. Am Neurol 1987;21: in-vitro

2 Edvinsson L, et al. Reduced levels of calcitonin gene-related peptide-like immunoreactivity in human brain vessels after subarachnoid haemorrhage. Neurosci Lett 1991;121:151-4.

PULSATILITY OF CEREBRAL BLOOD FLOW VELOCITY AS A FUNCTION OF AUTOREGULATORY RESERVE: EXPERIMENTAL STUDY IN NORMAL ANAESTHETIZED RABBITS M Czosnyka, HK Richards, PJ Kirkpatrick, JD Pickard. Addenbrooke's Hospital, Cambridge, UK

The pulsatility of cerebral artery blood flow velocity (FV) assessed by transcranial Doppler (TCD) ultrasonography is dependent on cerebral autoregulatory reserve. ${ }^{12}$ Why does the pulsatile component of the FV waveform increase with induced hypotension and how does TCD assessment of the autoregulatory threshold relate to laser Doppler (LDP) findings?

Eleven anaesthetized, ventilated rabbits ( $\left.\mathrm{PaCO}_{2}: \quad 30-38 \mathrm{mmHg}\right)$, had arterial blood 
pressure (ABP) monitored in the common carotid artery (pressure monitor, Camino Laboratories, United States), FV in the basilar artery was measured using $8 \mathrm{MHz}$ transcranial Doppler (PCDop, Scimed, UK) and LDP flux was obtained from the hemisphere contralateral to the side of insertion of the Camino probe (Laser Blood Flow Monitor, Moor Instruments, UK). All waveforms were recorded and analysed on the authors' own computer system under resting conditions and during haemorrhageinduced hypotension. Results were then standardized for $\mathrm{PaCO}_{2}$ and averaged.

Both average of flux and systolic FV started to decrease below an ABP of 45 mmHg; mean FV started to decrease gradually below $70 \mathrm{mmHg}$ with a more rapid fall below $45 \mathrm{mmHg}$. Diastolic FV fell rapidly below $70 \mathrm{mmHg}$. The difference between these thresholds ( 45 and $70 \mathrm{mmHg}$ ) was equal to the systolic-diastolic difference of ABP at a mean pressure of $50 \mathrm{mmHg}$. Standardised pulsatility index (SPIdefined as the Gosling pulsatility index divided by $\mathbf{A B P}_{\text {amplitude }}$ ) started to increase at ABP $70 \mathrm{mmHg}$, reaching a maximum at ABP $30 \mathrm{mmHg}$ and then fell. It was concluded that, in the healthy rabbit, the decrease in diastolic FV takes place earlier (by ABP pulse peak-to-peak value) than the exhaustion of autoregulatory reserve. This phenomenon is responsible for the early increase in SPI. Decrease in systolic FV marks the limit of autoregulation indicated using LDP flux.

1 Chan $\mathrm{KH}$, Miller JD, Dearden $\mathrm{M}$, et al. $\mathcal{f}$ Neurosurg 1992;77:55-61.

2 Nelson RJ, Czosnyka M, Pickard JD, et al. Neurosurgery 1992;31:705-10.

PROLONGED DERANGED CEREBRAL OXYGEN METABOLISM WITH TEMPORARY CAROTID CLAMPING

AHS Huneidi, A Bristow, F Afshar. St Bartholomew's Hospital, London, UK

Cerebral oxygen metabolic rate $\left(\mathrm{CMRO}_{2}\right)$ remains an evasive parameter of crucial importance. It can be calculated as the product of cerebral blood flow (CBF) and the arterio-jugular oxygen content difference $\left(\mathrm{AJDO}_{2}\right)$. This study shows a method for direct monitoring of $\mathrm{CBF}$ and cerebral tissue oxygen tension $\left(\mathrm{PcerO}_{2}\right)$ and $\mathrm{CMRO}_{2}$, and its significance in potential ischaemia with temporary arterial occlusion. $\mathrm{NZ}$ white rabbits (mean weight (SD) $2 \cdot 4$ $(0 \cdot 2) \mathrm{kg} ; \mathrm{n}=12$ ) were used for temporary bilateral common carotid artery occlusion. A Clarke's electrode (1 $\mathrm{mm})$, implanted in the cerebral cortex, continuously measured the $\mathrm{PcerO}_{2}$ and $\mathrm{CBF}$ was continuously recorded using a laser-Doppler shift monitor with an implantable dedicated cerebral fibreoptic catheter $(0.5 \mathrm{~mm})$. Recording was started 45 minutes before temporary bilateral carotid artery clamping (10 minutes) and continued for 120 minutes thereafter. Peripheral arterial $\left(\mathrm{PaO}_{2}\right)$ and jugular bulb $\left(\mathrm{PjO}_{2}\right)$ oxygen tensions were measured serially at regular intervals. The $\mathrm{PcerO}_{2}$ showed a good correlation with $\mathrm{PaO}_{2}$ and $\mathrm{PjO}_{2} \quad(\mathrm{r}=0.92$ and 0.94 , respectively; $\mathrm{p}<0.001)$ before carotid clamping. These correlations and the corresponding $\mathrm{CMRO}_{2}$ were not maintained during and after the temporary carotid artery occlusion.

It is concluded that temporary cerebral arterial occlusion can result in a prolonged
$\mathrm{CMRO}_{2}$ disorder. This technique can be a basis for cerebral blood flow and oxygen tension and $\mathrm{CMRO}_{2}$ monitoring at surgery and in the ventilated patients with cerebral vascular and traumatic lesions.

MINIMALLY INVASIVE SURGERY FOR TEMPORAL LOBE EPILEPSY: A TECHNIQUE FOR HIGHLY SELECTIVE MEDIAL TEMPORAL LOBECTOMY USING PEROPERATTVE IMAGE GUIDANCE AND ELECTROPHYSIOLOGY

D Sandeman, J Bird, H Morgan, S Butler. Frenchay Hospital and The Burden Hospital and Institute, Bristol, UK

The authors have recently set up an adult epilepsy surgery service in Bristol. In addition to the standard facilities for epilepsy assessment, they are in the unique position in Europe of having access to detailed peroperative physiology and frameless image-directed surgery. As a result they have adopted a minimally invasive approach to the surgery of temporal lobe epilepsy. The initial series of 21 operative procedures on 18 patients includes seven cases of selective medial temporal lobectomy. Three of these had a structural lesion on MRI or interictal single photon emission CT, while four had no structural abnormality demonstrable radiologically. The structural lesions were removed using the ISG viewing wand by the technique already described to the society. In the other cases, $2 \mathrm{~mm}$ axial reconstructions from a $\mathrm{T} 1$-weighted volume acquisition MRI were used to direct the viewing wand. The position of a $3 \mathrm{~cm}$ diameter temporal trephine was then determined using the wand. Specially constructed deep brain electrodes that attach to the wand were then passed across the middle temporal gyrus into the amygdala and the posterior hippocampus. Recordings from these and from subdural strip electrodes were then carried out with Brietal provocation to localise abnormal spike activity. The extent of resection of the medial temporal structures was then tailored to the electrophysiological findings, the wand being used to identify the inferior horn of the lateral ventricle to allow orientation within the temporal lobe and to determine the position of the medial surface of the temporal lobe, before biopsy and removal using the ultrasonic aspirator, leaving the medial temporal arachnoid intact. No complications with the technique have been encountered and preliminary results in terms of epilepsy control are encouraging. Preoperative and postoperative volumetric analysis is being carried out and these results were presented. The interesting and controversial implications of this approach were discussed.

VOLUMETRIC ANALYSIS OF EPILEPSY SURGERY RESECTIONS USING HIGH RESOLUTION MRI ND Kitchen, DGT Thomas, SD Shorvon, DR Fish, J Stevens. Institute of Neurology, London, UK

Epilepsy surgery is becoming increasingly guided by preoperative imaging, particularly MRI. Postoperative appearances, however, have not been studied to any extent. This is a significant omission as an accurate means of defining the surgical resection is essential to any meaningful follow-up outcome study. The authors therefore attempted to define the volume and anatomical site of epilepsy resections using high resolution MRI.

MRI was performed three months after surgery on a GE $1.5 \mathrm{~T}$ Signa unit. Volumetric imaging was performed in the coronal plane using contiguous T1 $1.5 \mathrm{~mm}$ slices. Sagittal T1 and axial T2 and proton density series were also obtained. Accuracy was assessed using postmortem studies. Variability within and between observers was consistently less than $5 \%$. The technique was applied to 50 patients following a wide variety of operations for temporal and extratemporal lobe epilepsy. Considerable variability in resection volume and anatomy was found which may be of considerable prognostic importance. Comparison with preoperative MRI has enabled the correlation of the site of resection with the preoperative abnormality. Postoperative volumetric MRI provides clinically useful information for individual patient management, and could prove invaluable for comparison of different epilepsy surgery techniques.

MULTIPLE SUBPIAL TRANSECTION: THE MAUDSLEY EXPERIENCE

IJA Robertson, IMS Swahney, CD Binnie, CE Polkey. Maudsley Hospital, London, UK

Multiple subpial transection (MST) is a novel technique in epilepsy surgery for those patients where the epileptogenic lesion cannot be resected because it is in an eloquent area. The first 14 patients to undergo MST by the authors were reviewed. Twelve patients had medically intractable epilepsy and two patients had Landau-Kleffiner syndrome. Their ages ranged from 8-47 (mean 17) years. Their duration of epilepsy $0 \cdot 75-42$ (mean 10) years. Preoperative MRI showed a focal abnormality in five cases. Detailed electrophysiological studies, including intraoperative electrocorticography were carried out in all cases. Most cases underwent MST of precentral and postcentral areas. In addition, five patients had brain resected and seven patients had a biopsy. Histopathology revealed five cases of Rasmussen's syndrome, four cases of cortical dysplasia and non-specific changes in three cases. Patients have been followed up for 6-48 months (mean 16). Eight patients have experienced marked reduction in seizure frequency, four patients have had limitation of seizure propogation. The two patients with LandauKleffner syndrome have shown very good recovery of language. No patient experienced significant additional neurological deficit.

FUNCTIONAL HEMISPHERECTOMY IN THE MANAGEMENT OF INTRACTABLE SEIZURES IN CHILDHOOD

W Harkness. Hospitals for Sick Children, London, UK

Between April 1992 and March 1993 functional hemispherectomy has been performed in six children at the Hospitals for Sick Children. Indications for surgery were severe intractable seizures in the presence 
of an established functional neurological deficit. Age of onset of seizures ranged from before birth to eight months before hemispherectomy and age at surgery was from two to nine years. Preoperative investigation included EEG, neuropsychology, and anatomical and functional imaging. Underlying pathology was a neuronal migrational defect in most cases, with one case of Rasmussen's encephalitis. Functional hemispherectomy was performed following intraoperative electrocorticography which demonstrated widely variable electrical abnormalities. The surgical technique was described and compared with other described techniques of hemispherectomy. Early results were discussed in terms of functional and seizure outcome.

TEMPORAL LOBECTOMY FOR INTRACTABLE EPILEPSY. EXPERIENCE WITH 58 CASES OVER 21 YEARS

KG Davies, RD Weeks. University Hospital of Wales, Cardiff, UK

Sixty patients with intractable complex partial seizures underwent surgery between 1969 and 1990, and 58 of these underwent a temporal resection. Of 39 patients who had chronic subdural electrocorticography (ECoG) 37 subsequently underwent an anterior temporal lobectomy (ATL) and two had the electrodes removed without resection. Two patients had bilateral depth electrodes placed and then had an ATL. Fourteen patients with evidence of temporal structural lesions had temporal resections with intraoperative ECoG, and five had resection without ECoG. Mean length of follow-up for all patients was six years. A total of $55 \%$ of cases were seizure free postoperatively, $7 \%$ almost seizure free, $21 \%$ had worthwhile improvement and $17 \%$ no improvement. The outcome for patients with structural lesions was particularly good. Nine patients complained of mild memory impairment postoperatively and one had a severe amnestic problem. One patient with an unsuspected tumour developed a hemiparesis and dysphasia. One other patient had persistent dysphasia. No mortality was attributable to the surgery. It is concluded that ATL is an excellent operation for the treatment of intractable epilepsy arising from the temporal lobe, and chronic subdural ECoG is a safe and reliable method for localising the seizure onset.

NEUROSTIMULATION FOR MOVEMENT DISORDERS

JC Sutcliffe, TH Koeze, ES Watkins. Royal London Hospital, London, UK

It has been known since the last century that neurostimulation, at least in animals, could be used to control muscle tone ${ }^{1}$ and this has become clinically significant. ${ }^{2}$ Involuntary movement disorders are notoriously difficult to treat, despite an increasing variety of drugs.

A series of 31 patients in whom a neurostimulator was implanted for a variety of movement disorders is presented and these patients were assessed in a simple manner, to answer the question: did the neurostimulator in any way improve their motor functions? This study revealed that all but one patient had improved function and reduced symptomatology after neurostimulation. Complications related to electrical failure (nine cases) and infection (six cases) were common, but did not prevent the patients seeking further intervention, because of the degree of benefit obtained.

It is suggested that neurostimulation is indicated in selected patients with involuntary movement disorders, to control their debilitating symptoms, when more conventional methods of management have failed.

1 Lowenthal J, Horsley V. Proc R Soc Lond 1897; 61:20.

2 Waltz JM, Andreesen WH, Hunt DP. Pacing Clin Electrophysiol 1987;10:180-204,

MAGNETIC RESONANCE ANGIOGRAPHY CAN IDENTIFY FIFTH NERVE COMPRESSION IN TRIGEMINAL NEURALGIA

JFM Meaney, JB Miles, E Ballantyne, TE Nixon, GH Whitehouse. Walton Centre for Neurology and Neurosurgery, Liverpool and University of Liverpool, Liverpool, UK

Magnetic resonance angiography (MRA) was performed in 16 patients with trigeminal neuralgia. The axial images were inspected to establish the exact relationship of the trigeminal nerve to the adjacent arteries. If a vessel was seen compressing the nerve, the angiogram was inspected to identify the vessel and its origin.

In 12 patients compression of the trigeminal nerve on the symptomatic side was identified by MRA. In 10 cases this was by a single vessel whereas, in the remaining cases, compression by two vessels was noted. The superior cerebellar artery was responsible for all cases in which compression was by a single vessel. In the two patients with double compression, the nerve was sandwiched between the anterior inferior cerebellar artery and superior cerebellar artery in one patient; and between the anterior inferior cerebellar artery and a persistent trigeminal artery in the other patient. Four patients had negative results.

In nine patients with positive MRA studies the findings were confirmed at posterior fossa exploration. Two of the patients with negative scans had previously undergone posterior fossa exploration which failed to reveal a cause for their continuing trigeminal neuralgia. Surgery was not offered to two patients because of the normal findings on MRA.

To date, there has been complete correlation between MRA and the surgical findings. MRA is proposed as the preferred diagnostic investigation in the preoperative assessment of patients with trigeminal neuralgia. It provides an added degree of confidence to patients and their surgeons, and also prevents unnecessary surgery.

PITFALLS IN THE DIAGNOSIS OF BLOCKED

CEREBROSPINAL FLUTD SHUNTS: A

PROSPECTIVE STUDY OF REFERRAL TO A PAEDIATRIC NEUROSURGICAL UNIT

LD Watkins, U Andar, WF Harkness, RD Hayward. Hospitals for Sick Children, London, UK

The diagnosis of shunt blockage can be a simple one when the patient presents with the classic symptoms and signs of raised intracranial pressure, and cranial scanning reveals an increase in the size of the ventricular system. In children, however headache, vomiting, drowsiness, irritability, etc may be associated with many other disorders (intercurrent infections, for example) and this may make the diagnosis of shunt failure less obvious, particularly to those non-neurosurgical specialists whose opinions may be the first to be sought by the patient's family.

At the Hospitals for Sick Children, Great Ormond Street, we studied prospectively all recent cases referred to the neurosurgical unit with a diagnosis of shunt blockage. Such children may have been brought in by their parents without previous medical consultation, or may have been referred by general practitioners or local paediatric units Our analysis revealed the relative accuracy of each of these three groups in making the diagnosis and identifies those conditions with which shunt failure is most likely to be confused. It also posed the question as to how accurately neurosurgeons should expect the diagnosis to be made in the community if a decline in the number of "false alarms" is not to be offset by even the slightest rise in the number of cases where failure to recognise the early symptoms of shunt blockage may result in serious neurological damage or even death.

THEORETICAL AND PRACTICAL ISSUES IN FRAME-BASED AND FRAMELESS STEREOTACTIC SURGERY PLANNING

L Lemieux, ND Kitchen, DGT Thomas. Institute of Neurology, London, UK

Stereotaxy is an ensemble of techniques for the accurate localisation of structures within a body through external means. To a large extent, stereotaxy has been synonymous with "CT stereotaxy", based on rigid frames attached to the patient's skull. Whereas originally only one CT slice could be analysed at a time, recent advances in computing have allowed complete data sets to be analysed simultaneously. This has also allowed the development of so-called frameless (landmark-based) stereotaxy. The fundamental theoretical and practical differences between the two approaches are discussed. Novel methods, based on perturbation studies, are presented for the estimation of the effect of stereotactic fiducial marker localisation on target coordinate calculation in a stereotactic surgery planning program, ${ }^{1}$ both for CT and digitised radiography. A method is introduced for the evaluation of the accuracy of stereotactic correlation of tomographic data which is based on the calculation of a volume transformation matrix (VTM). Results of the perturbation studies clearly indicate the necessity of sub-pixel registration of the fiducial markers in CT. The loss of accuracy associated with the use of the VTM indicates possible limitations in the accuracy of localisation based on frameless stereotaxy.

1 Lemieux L, Lester S, Fish DR. Electroencephalogr Clin Neurophysiol 1992;82:399-407.

THE VALUE OF STEREOTACTIC BRAIN BIOPSY IN PAEDIATRIC NEUROSURGERY

DA Jellinek, $M$ Vloeberghs, $G$ Turner, $K$ Robson, JAG Punt. Queens Medical Centre, Nottingham, UK 
A series of 26 paediatric image-guided, stereotactic brain biopsies was presented. The age range was $2-15$, mean 8.8 years. Twenty-four procedures were CT guided, whereas two were MRI guided. The lesion was hemispheric in seven cases, parasellar in one case, pineal in one, located in the diencephalon/brainstem in 15, and cerebellar in two patients. There was no morbidity or mortality after the procedures.

The diagnostic confidence and correlations of the preoperative clinical and radiological diagnoses were reviewed in comparison with the pathological diagnosis (frozen section and definitive specimen histology). In most patients the preoperative management plan was confirmed by the histological diagnosis. In three key cases, of cerebral lymphoma, cerebral vasculitis, and primitive neuroectodermal tumour, however, the histological diagnosis radically altered management.

It is concluded that stereotactic biopsy in children is technically feasible, safe, and is a major contributor to the management of paediatric neurosurgical disease.
DOES STEREOTACTIC BRAIN BIOSPY IN PATIENTS WITH AIDS CONTRIBUTE TO THEIR MANAGEMENT?

R Viswanathan, J Ironside, JE Bell, RP Brettle, IR Whittle. Western General Hospital, Edinburgh, UK

Twelve lesions have been biopsied in nine patients who were either HIV positive or had AIDS. Indications for biopsy were clinical deterioration despite active medical management in HIV positive patients with cerebral lesions. Preoperatively most patients were thought to have toxoplasma, CNS lymphoma or progressive multifocal leukoencephalopathy (PML). Most patients had signs of focal CNS dysfunction and psychomotor slowing. Mean CD4 count was 45 (normal > 500). Biopsy was performed using CT imaging and a BRW stereotactic system. One biopsy revealed non-specific gliosis and microcalcification but the others showed toxoplasmosis (four), CNS lymphoma (three), PML (three), HIV encephalitis (two) and one case of non-specific encephalitis. In $25 \%$ of the specimens, single biopsy specimens revealed more than one pathology-for example, contiguous CNS lymphoma and toxoplasma, HIV encephalitis and toxoplasma. Complications of biopsy included one delayed (eight days) biopsy site haematoma and one incidental subarachnoid haemorrhage but neither required any special treatment. Median survival after biopsy was seven weeks. Although the impact of tissue diagnosis on patient survival was poor, it did enable modification of drug treatment such as cessation of toxoplasma therapy, commencement of antiviral therapy for PML, and treatment-limiting decisions to be made-for example, withdrawal of treatment in patients with CNS-lymphoma or HIV encephalitis.

The conclusions of this study are firstly that the outcome is uniformly poor, irrespective of pathology, in patients who are HIV positive and in a bad clinical and immunological state at the time of biopsy. Secondly with comprehensive neuropathological studies, the diagnostic yield from stereotactic biopsy specimens is similar to non-AIDS cases. Although histological diagnosis may facilitate decisions affecting quality of life in these patients, whether earlier biopsy would alter life expectancy is uncertain. 